\title{
Design of Cortical Neuron Circuits With VLSI Design Approach
}

\author{
A.D.Tete ${ }^{1}$ and Dr. A.Y.Deshmukh ${ }^{2}$ \\ ${ }^{1}$ Research scholar, G.H.Raisoni College of Engg., Nagpur \\ ${ }^{2}$ Head, Dept.of Electronics G.H.Raisoni College of Engg., Nagpur \\ ${ }^{1}$ aruna.mathurkar@gmail.com, ${ }^{2}$ aydeshmukh@gmail.com
}

\begin{abstract}
A simple CMOS circuitry using very less number of MOSFETs reproduce most of the electrophysiological cortical neuron types and is capable of producing a variety of different behaviors with diversity similar to that of real biological neuron cell. The firing pattern of basic cell classes like regular spiking (RS), chattering $(\mathrm{CH})$, intrinsic bursting $(\mathrm{IB})$ and fast spiking $(F S)$ are obtained with a simple adjustment of only one biasing voltage makes circuit suitable for applications in reconfigurable neuromorphic devices that implement biologically resemble circuit of cortex. This paper discusses spice simulation of the various spiking pattern ability with required and firing frequency of a given cell type. The circuit operation is verified for both conditions-constant input and pulsating input.
\end{abstract}

\section{KEYWORDS}

Neocortex, Dendritic morphology, Oscillatory Neuron, Cortical neuron

\section{INTRODUCTION}

The neocortex is that part of the brain which makes up the outer 2 to $4 \mathrm{~mm}$ of the cerebral hemispheres. It is the "gray matter" of the brain lying atop the cerebral "white matter' composed of myelinated axons that interconnect different regions of the brain. All the higher-level psychophysical functions sensory perception, object and event representation, planning and decision making are believed to take as their biological substrate the activities of interconnected and distributed networks of neurons in the neocortex. The cortex structure is very thin and highly folded with many grooves. All sensory information reaching the neocortex is conveyed through a sub-cortical (below the cortex) structure called the thalamus. Other signals, thought to be primarily 'control' signals that modulate cortical activity, also come into the neocortex from approximately 20 sub-cortical regions of the brain. Different regions of the neocortex appear to be specialized to participate in specific type of psychophysical functions[12]. No single area of the brain has been successfully identified as the sole functional area of any psycho-physical phenomenon. 
The basic component of the cortical microcircuits are neuron cells. Mimicking it's operation in silicon circuits is a subject of ongoing research interest. Analog VLSI model(Very Large Scale of Integration) of neural circuits provides efficient emulation engines. This could lead in developing the electronic devices that mimic the operation of biological brain. This ongoing research will make it possible to make more realistic model of human brain. These models are important tools for characterizing what nervous system do, determining how they function, and understanding why they operate in particular ways.

Power consumption and area required to design certain model with least number of transistor is a fact of adopting the VLSI neural networks that consists of many thousands of neurons.

Growing interest approaches towards the spike-based neural network as they appears to provide promising solution to a variety of complex problems which can not be solved by most powerful computers. This paper focuses on the different spiking and firing patterns of the cortical neuron. Typically used I\&F (Integrate \&Fire) cells consume approximately 20 transistors to implement low power adaptive neuron circuitry [4][5]. This is not capable of imitating the processing of human nervous system, as approximately $90 \%$ of the cortex is made of non-linear oscillatory neuron rather than simple spiking neurons. Therefore work focuses on the utilization of the minimum number of transistors providing different types of firing patterns obtaining different types of adaptive and oscillatory neuron behaviors in a single chip.

The feature of biological neural network are attributed to it's structure and function. Fundamental unit of the network is called a neuron or a nerve cell. It consists of a cell body or soma where the cell nucleus is located. Tree like nerve fibers called dendrites are associated with the cell body. These dendrites receives signals from other neurons. Extending from the cell body is a single long fiber called the axon, which branches into strands and sub strands connecting to many other neuron at the synaptic junctions or synapses.

Generally the electrical activity is confined to the interior of a neuron, whereas the chemical mechanism operates at the synapses. The dendrites serve as receptors for signals from other neurons, whereas the purpose of an axon is transmission of the generated neural activity to other nerve cell or to muscle fibre..

Artificial neural network (ANN) is a highly simplified model of the structure of the biological neural network. ANN consists of interconnected processing units. The general model of a processing unit consist of summing part followed by output part. The summing part receives u1,u2----un input values(w1,w2----wn) and computes a weighted sum. The weighted sum is called the activation value. The output part produces a signal from activation value. The sign of the weight for each input determines whether the input is excitatory(positive weight) or inhibitory(negative weight). The input could be discrete or continuous data values, and likewise the output also be deterministic or stochastic or fuzzy.

In an artificial neural network several processing units are interconnected according to some topology to accomplish a pattern recognition task. Therefore the input to a processing unit may come from the output of other processing units, and/or from external sources. The output of each units may be given to several units including itself. The amount of the output of one unit received by another unit depends on the strength of the connection between the units and it is reflected in the weight value associated with the connecting links. If there are $\mathrm{n}$ activation value of the network defines the activation state of the network at that instant. 
International Journal on Soft Computing ( IJSC ) Vol.2, No.4, November 2011

Neurons in the cortex are found with great variety of dendritic morphology, ion channel distribution and composition. Hence these neurons exhibit different electrical behavior transforming the same input signal into different firing patterns. Many parameters such as spike frequency, inter spike interval histogram, spike frequency, adaptation index etc. can be used to classify the neurons. Approximately $90 \%$ of the cortex is made up of non-oscillatory neuron rather than simple spiking neurons.

Therefore effort has been made for implementing simple neuron circuits that are capable of providing different types of cortical spiking behavior by utilizing as few transistors as possible to enable integration of large number of cell in a single chip. The neuronal response to a step stimulus of supra-threshold current (post synaptic input current that causes action potential) displays either spiking or bursting firing behavior. The spiking neurons are of two types like regular spiking (RS) and fast spiking (FS). The RS cell exhibit accommodation property. In response to a supra-threshold current step they fire repeatedly. The RS cell class can be further sub-divided into two sub-types-weak accommodating cell are RS1 and strong accommodating cell are called RS2. Pyramidal cells are an example of RS1 type and and stellate cells are an example of RS2 type. The FS cells fire repetitively at high frequency with little or negligible accommodation to a sustained supra-threshold current injection.

The action potential of FS cells exhibit faster rise and fall rates and distinct fast afterhyperpolarization (AHP) e.g. neocortical small basket cells, bitufted cells and large basket cells. The basic bursting cell types are chattering $(\mathrm{CH})$ and intrinsic bursting (IB). The $\mathrm{CH}$ neurons usually display repetitive long clusters of spikes. The IB neurons respond to a step current injection with a cluster of three to five initial spikes followed by an AHP, and then by either single spikes or burst at more or less regular intervals. These types are observed in sub-population of bitufted cells, bipolar cells and martinotti cells in the neocortex.

\section{VLSI MODEL OF CORTICAL NEURON}

Cortical microcircuits are capable of performing sophisticated information processing, handling high computational throughput of sensory perception, cognitive processes, control and decision making with low energy consumption. The basic component of the cortical microcircuits are neuron cells. Mimicking their operation in silicon circuit is the aim of the paper. It is hoped that analogue VLSI model of neural circuits will provide very efficient brain-inspiredcomputer architecture. It is an important consideration to design a neuron circuit with the least number of transistors and with least energy consumption, especially due to the fact that such circuit is intended to be used in large scale VLSI neural networks that consists of many thousands of neuron. While considering presently available neuron models the integrate and fire (I\&F) neuron model is widely used due to it's simplicity - typical I\&F neuron cells use approximately 20 transistors to implement low power adaptive neuron circuitry. 


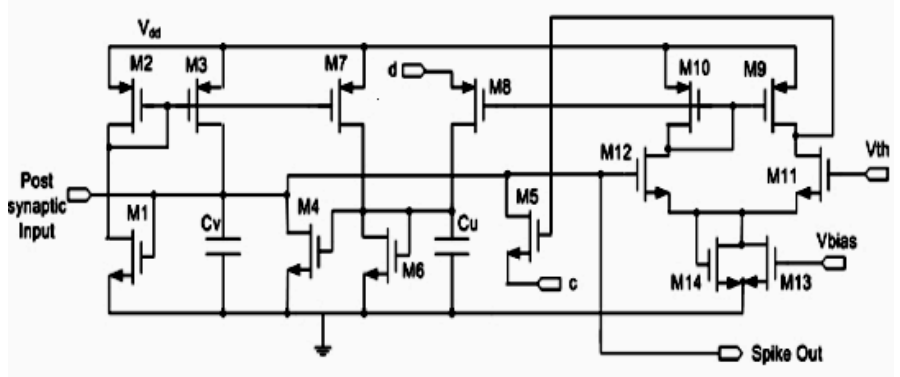

Fig.1 Circuit of cortical neuron

However, I \& F neurons exhibit simple firing behavior only, this might not be adequate for the development of VLSI circuitry which would be capable of imitating the processing of the cortex, which is made up of a large number of more complex non-linear oscillatory neurons exhibiting a variety of inherent firing patterns. Circuit implementing conductance based neuron model (Hodgkin-Huxley) consume large number of transistors. The circuit implementation of oscillatory neuron model Fitzhugh-nagumo, resonate and Fire model, Hindmarsh-Rose uses around 20 transistors. However all these models donot accurately reproduce the shapes of the spikes that have been observed in biological neurons and are also not capable of generating different types of spiking and bursting behavior in a single circuit with tunable parameters.

The work presents a simple CMOS circuit model that exploits underlying non-linear characteristics of MOSFETs to implement the neuron using only 14 transistors. The spiking shape given by the circuit resembles that of real neurons. The circuit is capable of producing linear and non-linear responses (firing rate vs. input current) with spike frequency adaptation and a variety of spiking patterns such as regular spiking, fast spiking, low threshold spiking, intrinsic bursting etc.

The proposed silicon cortical neuron circuit contains 14 MOSFETs. The two state variables "membrane potential" (v) and "slow variable" (u), are represented by voltages across capacitors $\mathrm{Cv}$ and $\mathrm{Cu}$ respectively. The circuit consists of three functional blocks: membrane potential circuitry, slow variable circuitry and comparator circuitry.

\subsection{Membrane Circuit}

Fig. 2 - illustrates the membrane potential circuit where the magnitude of the current through M3, Iv, is controlled by the membrane potential V. Transistors M2 and M3 form a current mirror circuits, with input current generated by M1. The current Iv acts as a positive feedback to generate spikes.The current Il is the leakage current generated by M4 and is controlled by the slow variable $U$. The current I is the post synaptic input current and it is supplied by an external synapse. The net sum of these currents is integrated on the membrane capacitor $\mathrm{Cv}$ : 


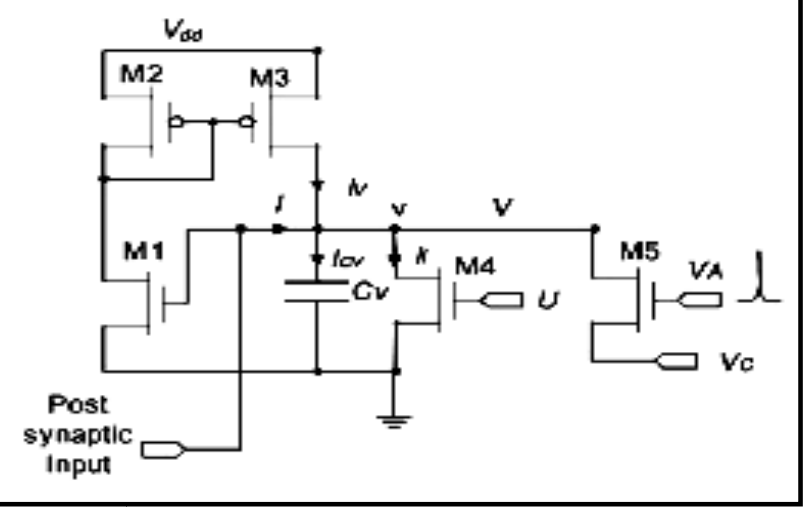

Fig.2 Membrane circuit

Positive input current leads to the increase in $\mathrm{V}$, which becomes more rapid as $\mathrm{V}$ increases, generating the spike. Once the spike is detected (by the comparator circuit) a pulse on VA is generated. Consequently transistor M5 opens and membrane potential voltage is rapidly hyperpolarized. The transistor M5 is designed so that the capacitor $\mathrm{Cv}$ is fully discharged during the VA pulse, thus the value of $\mathrm{V}$ after hyper-polarization is entirely determined by the value set by the voltage Vc.

\subsection{Slow Variable Circuit}

The magnitude of the current provided by M7, Ivu is determined by the membrane potential. Transistor M2 and M7 form a current mirror circuit with input current generated by M1. The transistors are scaled so that the drain current of transistor M7 is lower than that of M3 and capacitance value of $\mathrm{Cu}$ is selected as larger than that of $\mathrm{Cv}$. This ensures that potential $\mathrm{U}$ will vary more slowly than $\mathrm{V}$.

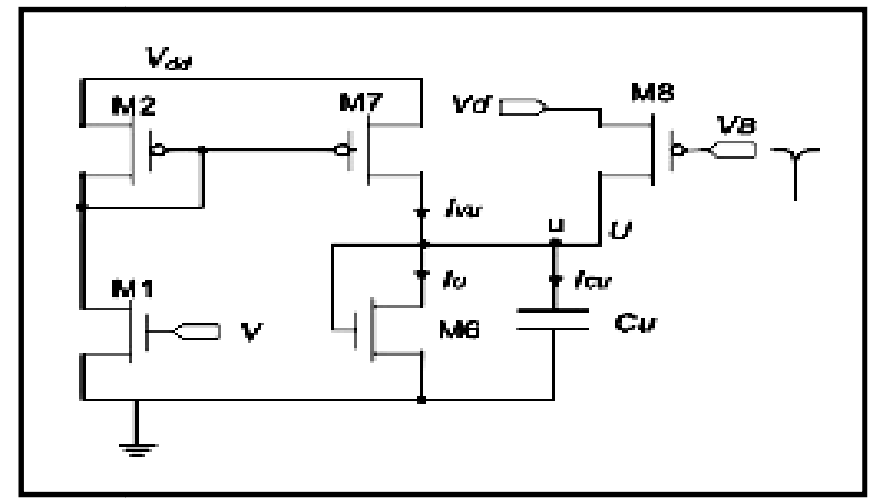

Fig.3 Slow variable circuit

The transistor M6 operates as a non-linear resistor and the current through M6 Iu is a function of the slow variable potential U. The net sum of these currents is integrated on the slow variable capacitor $\mathrm{Cu}$.

Following the membrane potential spike, the comparator generates a pulse, VB to open the transistor M8, The narrow size of M8 and short duration of pulse VB ensures that the capacitance 
$\mathrm{Cu}$ is not fully reset to $\mathrm{Vd}$, but instead an extra amount of charge, controlled by $\mathrm{Vd}$, is transferred onto $\mathrm{Cu}$. Therefore each membrane spike provides a quick increase in the slow variable potential which in turn increases the leakage current of the membrane potential and slow down the depolarization after the spike. This mechanism is used to provide the accommodation property of the spike train.

\subsection{Comparator}

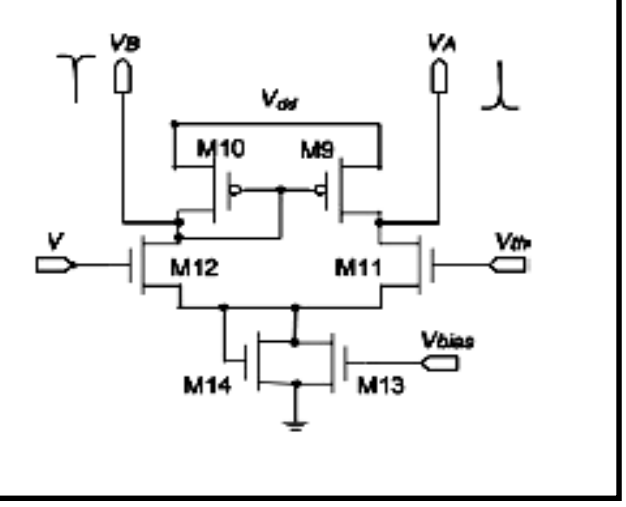

Fig.4 Comparator circuit

The comparator circuit is shown in Fig. 4. The voltage Vth is the spike-detection threshold of the membrane potential. The voltage Vbias controls the bias current in the comparator. When the membrane potential increases above Vth, the voltage at VB is decreased and VA is increased, generating reset signals. Due to limited speed of comparator and switches the reset is delayed, so the membrane potential V continues to increase beyond Vth, and onto VDD, but once VA is increased, the membrane potential is reset to $\mathrm{Vc}$ which is lower than Vth. Consequently, voltages VA and VB return to their reset voltage level, completing reset pulse. The transistor M14 increases the comparator current during the spike, providing the required amplitude and duration of the reset pulse VB.

\section{SIMULATION RESULTS}
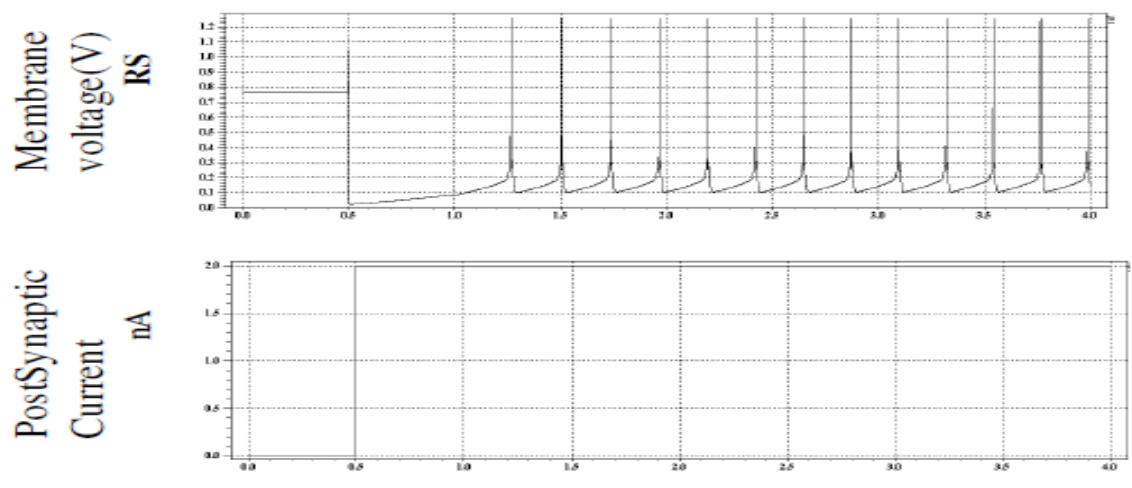

Time (ms)

Fig .3.1 Regular spiking pattern(RS) 
As the name suggests Regular Spiking (RS) gives a single spike output repeated at regular intervals. In the result shown above the spike repetition rate is once every $0.25 \mathrm{~ms}$. which in terms of frequency is $4 \mathrm{KHz}$. The step input for this simulation is $2 \mathrm{nA}$. It is seen that the firing pattern of VLSI neuron are on the millisecond scale of biological neuron. Regular spiking pattern Vd to be set to $2.07 \mathrm{~V}$. Threshold voltage at the comparator stage of the cortical neuron is set to $1.4 \mathrm{~V}$ and the bias voltage $\mathrm{Vc}$ is set to $0.6 \mathrm{~V}$ which is kept constant for all firing patterns . Single spike is necessary for the neuron to fire. It is generally observed in the mammalian neocortex.
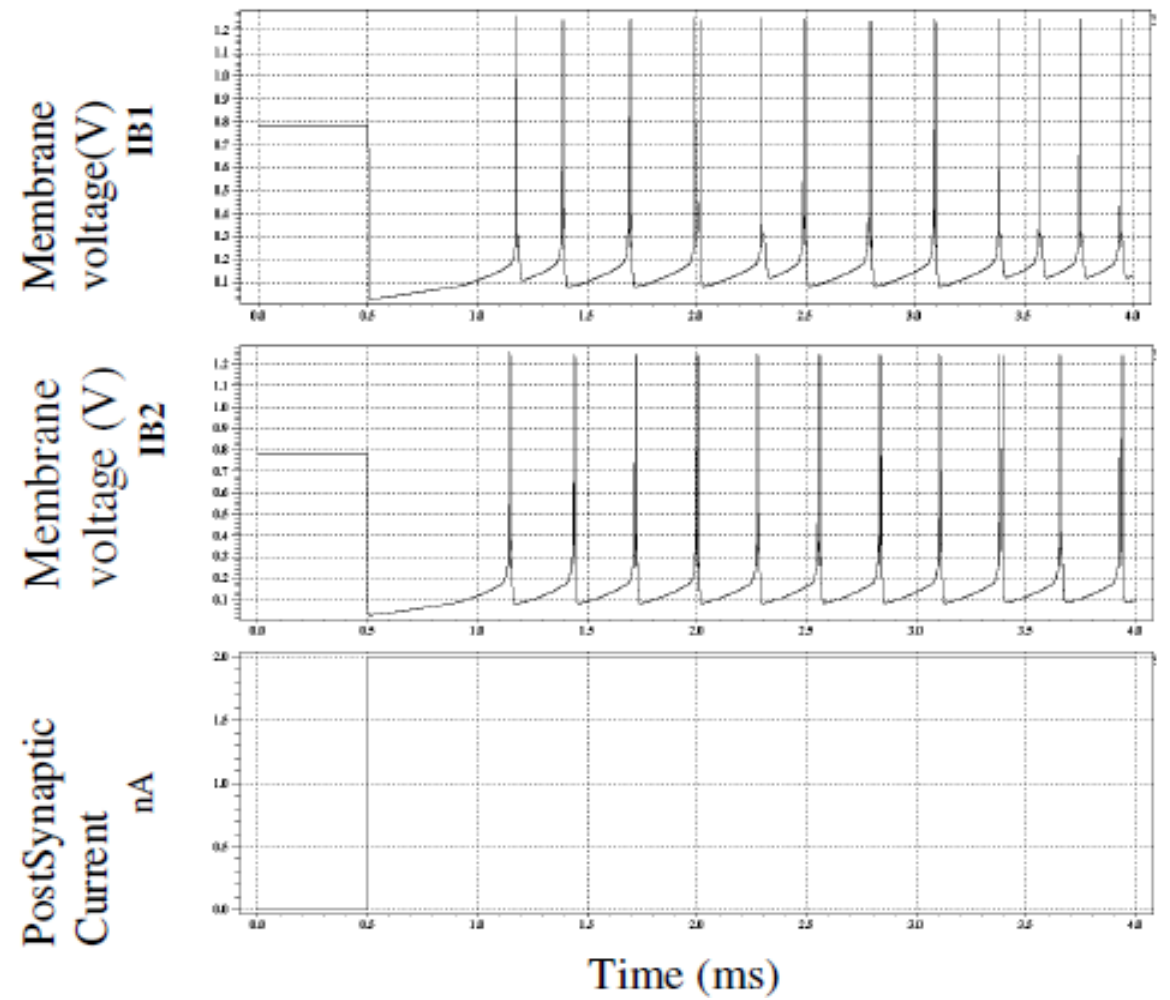

Fig.3.2 Intrinsic bursting spiking pattern(IB1 andIB2)

Intrinsical Bursting (IB) is a pattern in which more than one spikes or a Burst is repeated at regular intervals. Top trace of Fig. 3.2 shows the output obtained when Vd is kept at 2.02 V while the trace below it is for $\mathrm{Vd}=2.0 \mathrm{~V}$, the input remaining the same in both the cases. Once again the spike burst repetition rate is once every $0.25 \mathrm{~ms}$, which in terms of frequency is $4 \mathrm{KHz}$. The step input for this simulation is $2 \mathrm{nA}$. It is observed that when the $\mathrm{Vd}$ is 2.02 there is mixed type of spiking burst as well as single spike, this kind of patterns generally observed in the mammalian neocortex. 

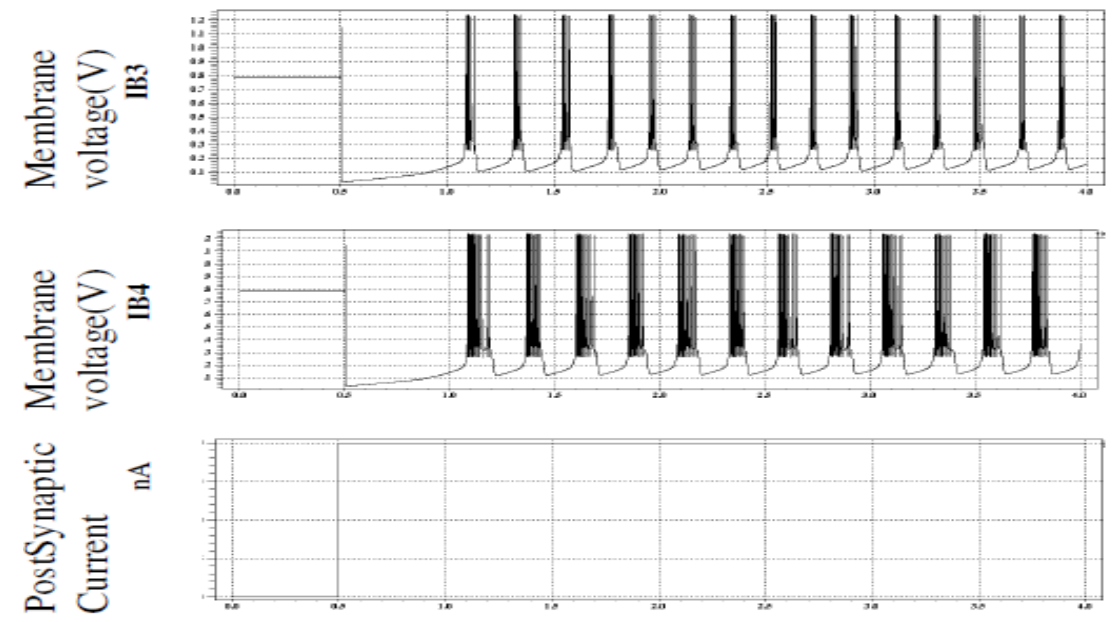

Time (ms)

Fig.3.3 Intrinsic bursting spiking pattern(IB3 and IB4)

Intrinsical Bursting (IB3 and IB4) is a pattern in which more than two spikes or a Burst is repeated at regular intervals. Top trace of Fig. 3.3 shows the output obtained when $\mathrm{Vd}$ is kept at $1.6 \mathrm{~V}$ while the trace below it is for $\mathrm{Vd}=1.4 \mathrm{~V}$, the input remaining the same in both the cases. Once again the spike burst repetition rate is once every $0.25 \mathrm{~ms}$, which in terms of frequency is $4 \mathrm{KHz}$. The step input for this simulation is $2 \mathrm{nA}$. It is observed that as $\mathrm{Vd}$ goes on decreasing more number spikes are required to fire the neuron. Some neurons such as the chattering neuron in cat neocortex fire periodic burst of spikes when simulated. The interburst (between burst) frequency is very high and it is believed that such neurons contributes to the gamma frequency oscillation in the brain.
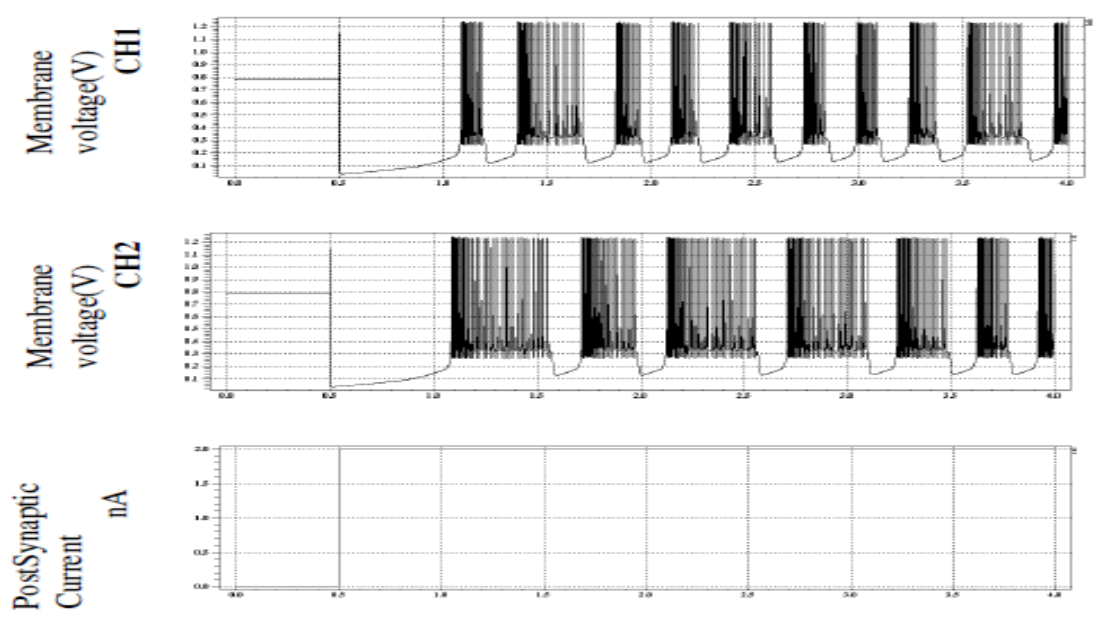

Time (ms)

Fig. 3.4 Chattering type spiking pattern $(\mathrm{CH} 1$ and $\mathrm{CH} 2)$

Chattering type ( $\mathrm{CH} 1$ and $\mathrm{CH} 2)$ is a pattern in which more than five to ten spikes or a Burst is repeated at irregular intervals. Accommodation period depend upon the number of spike required to fire the neuron. Top trace of Fig. 3.4 shows the output obtained when $\mathrm{Vd}$ is kept at $1.39 \mathrm{~V}$ 
while the trace below it is for $\mathrm{Vd}=1.35 \mathrm{~V}$, the input remaining the same in both the cases. Once again the spike burst repetition rate is once every $0.5 \mathrm{~ms}$ which in terms of frequency is $2 \mathrm{KHz}$. The step input for this simulation is $2 \mathrm{nA}$. It is observed that as $\mathrm{Vd}$ goes on decreasing more number spikes are required to fire the neuron. The interburst (between burst) frequency is very high.

Fast spiking cell fire repetitively at high frequency with less or no accommodation to a sustained suprathreshold current. Step input of $2 \mathrm{nA}$ is given to the cortical neuron with Supply voltage is set at $3 \mathrm{~V}$. Threshold voltage for comparison is set at $1.4 \mathrm{~V}$ while biasing is $0.4 \mathrm{~V}$. The control voltage $\mathrm{Vd}$ is set to $1.3 \mathrm{~V}$ for top trace and $1.0 \mathrm{~V}$ for later trace. As $\mathrm{Vd}$ is decreased to sufficient low level compared to RS pattern it is observed that there is no accommodation for the spikes that capacitor $\mathrm{Cu}$ does not get sufficient time to get discharge and at the output fast spiking pattern is observed.

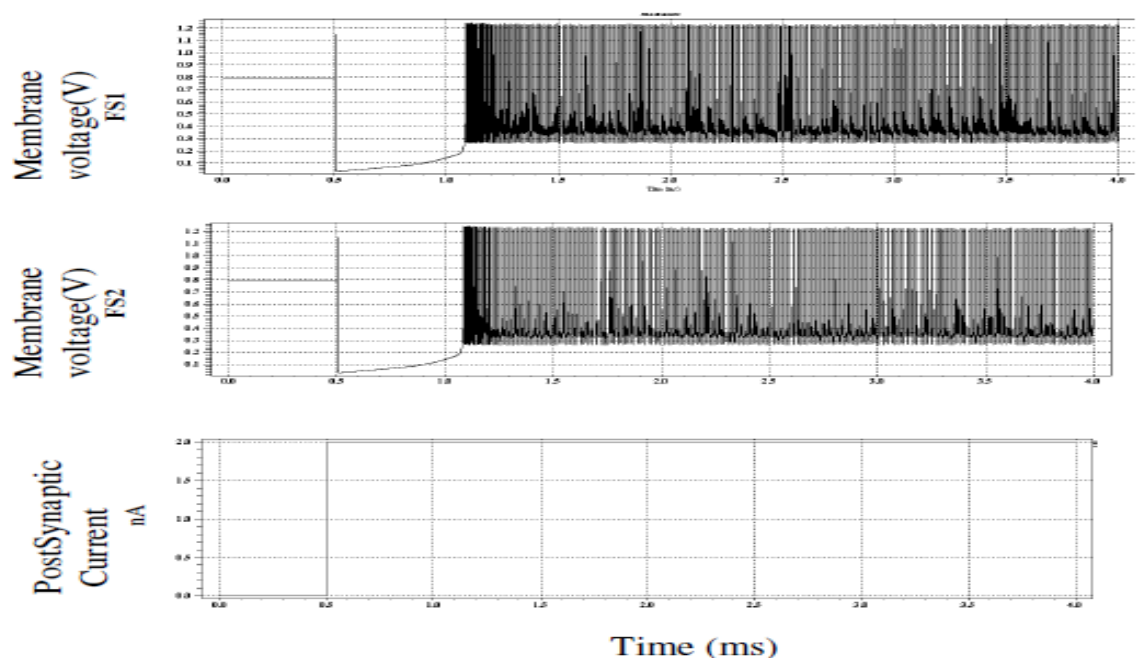

Fig.3.5 Fast spiking pattern(FS1 and FS2)

\begin{tabular}{|c|c|c|c|l|}
\hline Vd(V) & Ve(V) & $\begin{array}{l}\text { Spiking } \\
\text { Pattern }\end{array}$ & Delay(ms) & $\begin{array}{l}\text { Spiking } \\
\text { repetition } \\
\text { rate(freq) }\end{array}$ \\
\hline 2.07 & 0.6 & RS & 1.25 & $4 \mathrm{KHz}$ \\
\hline $2 . .02$ & 0.6 & IB1 & 1.1 & $4 \mathrm{KHz}$ \\
\hline 2.0 & 0.6 & IB2 & 0.55 & $4 \mathrm{KHz}$ \\
\hline 1.6 & 0.6 & IB3 & 0.55 & $4 \mathrm{KHz}$ \\
\hline 1.4 & 0.6 & IB4 & 0.5 & $4 \mathrm{KHz}$ \\
\hline 1.39 & 0.6 & CH1 & 0.5 & $4 \mathrm{KHz}$ \\
\hline 1.35 & 0.6 & CH2 & 0.5 & $2 \mathrm{KHz}$ \\
\hline 1.3 & 0.6 & FS1 & 0.1 & Continuous \\
\hline 1.0 & 0.6 & FS2 & 0.1 & Continuous \\
\hline
\end{tabular}

Table 1. Comparison of various spiking patterns of Cortical Neuron 
International Journal on Soft Computing ( IJSC ) Vol.2, No.4, November 2011

The circuits have been designed in a 1.25um CMOS technology. The simulation results of the circuits illustrates various types of cortical neuron firing patterns, obtained by changing the value of circuit variable $\mathrm{Vd}$ keeping $\mathrm{Vc}$ constant which are externally controllable. It is observed that with decreasing $\mathrm{Vd}$ the amount of charge transferred to slow variable capacitor decreases. Thus the capacitor charging time increases. The time till the capacitor voltage value reaches the threshold level fast spikes are continuously available at the output. Therefore decreasing $\mathrm{Vd}$ causes longer period of spikes and shorter duration of spike off or reset. Eventually at $\mathrm{Vd}=1.3$ the capacitor no longer reaches the threshold value and hence the reset due to slow variable does not occur. This causes continuous spikes to be available at the output. The patterns of VLSI neurons are on the millisecond scale of biological neurons. For comparative purposes, all the classifications are done in the time scaled domain in order to adopt biological classification method.
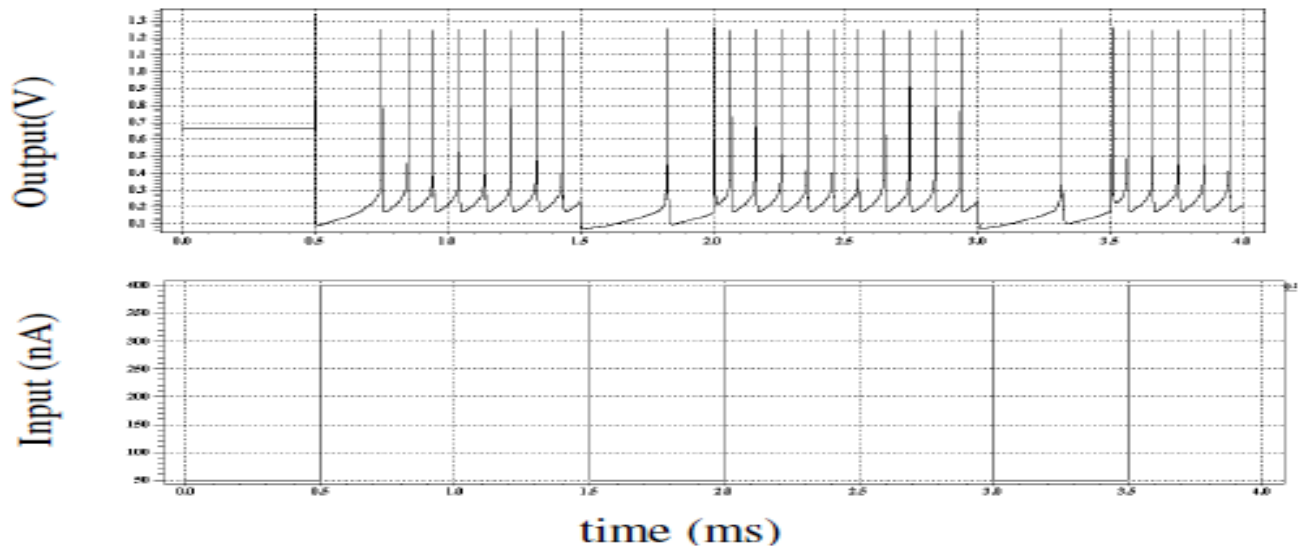

Fig 3.6 Response of amplitude variation on the regular spiking of cortical neuron

Another aspect of cortical neuron has been studied with the variation of the amplitude of the input signal. It is observed that amplitude of input signal (post synaptic signal) does influence the spiking pattern. An increase in input current not only causes an increase in spike repitition rate but also influences the slow variable circuit capacitor charging time. Thus the output pattern is different than the one with constant input current.

At the input side of Cortical neuron step of 400nA and 50nA are given with the threshold voltage $1.4 \mathrm{~V}$ and supply voltage $3 \mathrm{~V}$. It is observed that for $400 \mathrm{nA}$ the frequency of spiking pattern is 10 $\mathrm{KHz}$ while the frequency of spiking pattern for $50 \mathrm{nA}$ is $5 \mathrm{KHz}$. For both the input control voltage $\mathrm{Vd}$ is set to $2.07 \mathrm{~V}$.

It is also observed that delay for lower amplitude is more than that for higher amplitude. The delay for $400 \mathrm{nA}$ is $0.25 \mathrm{~ms}$ while delay for $50 \mathrm{nA}$ is $0.35 \mathrm{~ms}$. 

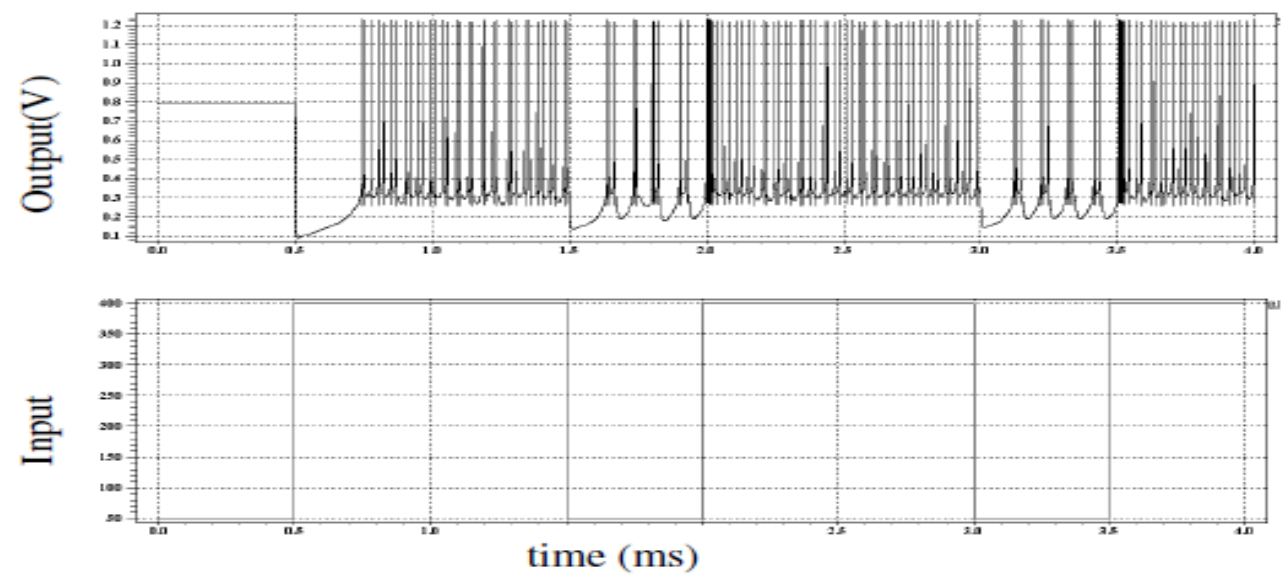

Fig 3.7 Response of amplitude variation on the intrinsic bursting of cortical neuron

Another spiking pattern (CH2 and IB2) for different amplitude and different control voltage is studied. At the input side of Cortical neuron step of 400nA and 50nA are given with the threshold voltage $1.4 \mathrm{~V}$ and supply voltage $3 \mathrm{~V}$. It is observed that for $400 \mathrm{nA}$ the frequency of spiking pattern is high while the inter-spike frequency of spiking pattern for $50 \mathrm{nA}$ is $6.6 \mathrm{KHz}$. For both the input control voltage $\mathrm{Vd}$ is set to $2.0 \mathrm{~V}$. It is also observed that delay for lower amplitude is less than that for higher amplitude. The delay for $400 \mathrm{nA}$ is $0.25 \mathrm{~ms}$ while delay for $50 \mathrm{nA}$ is $0.15 \mathrm{~ms}$.

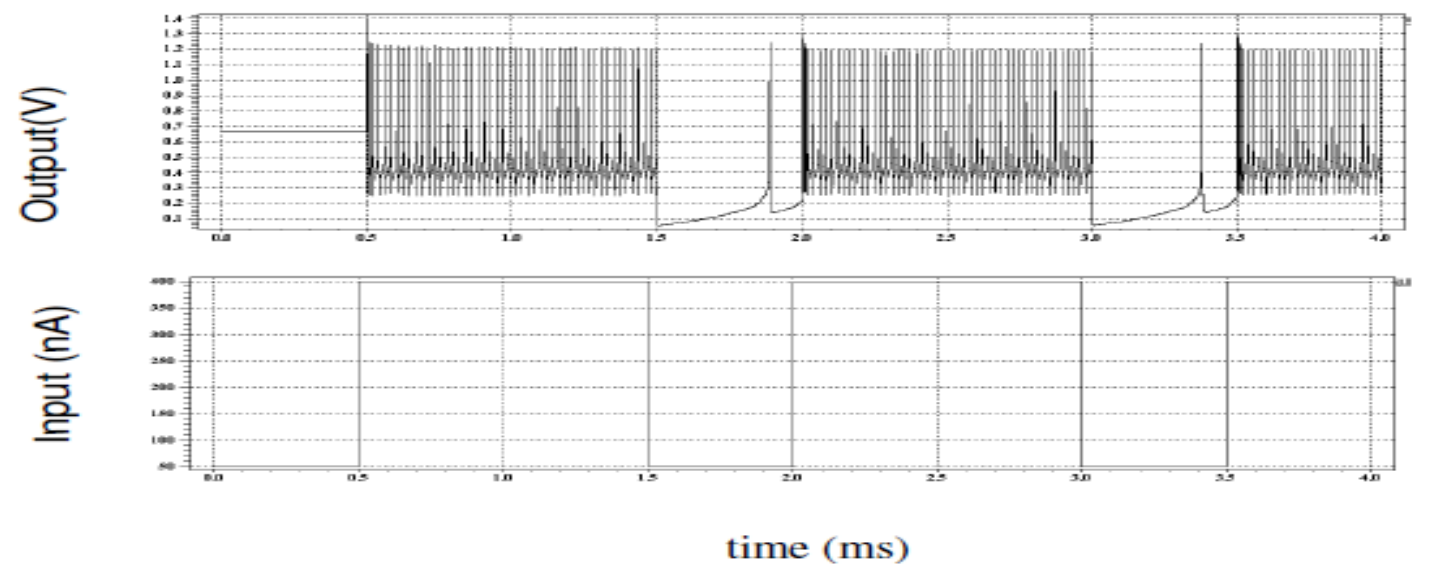

Fig 3.8 Response of amplitude variation on the fast spiking (FS1) of cortical neuron

Another spiking pattern (FS1 and RS1) for different amplitude and different control voltage is studied. At the input side of Cortical neuron step of 400nA and 50nA are given with the threshold voltage $1.4 \mathrm{~V}$ and supply voltage $3 \mathrm{~V}$. It is observed that for $400 \mathrm{nA}$ the frequency of spiking pattern is high while the spike frequency of spiking pattern for $50 \mathrm{nA}$ is $6.6 \mathrm{KHz}$. For both the input control voltage $\mathrm{Vd}$ is set to $1.39 \mathrm{~V}$.

It is also observed that delay for lower amplitude is more than that for higher amplitude. The delay for $400 \mathrm{nA}$ is $0 \mathrm{~ms}$ while delay for $50 \mathrm{nA}$ is $0.4 \mathrm{~ms}$. 

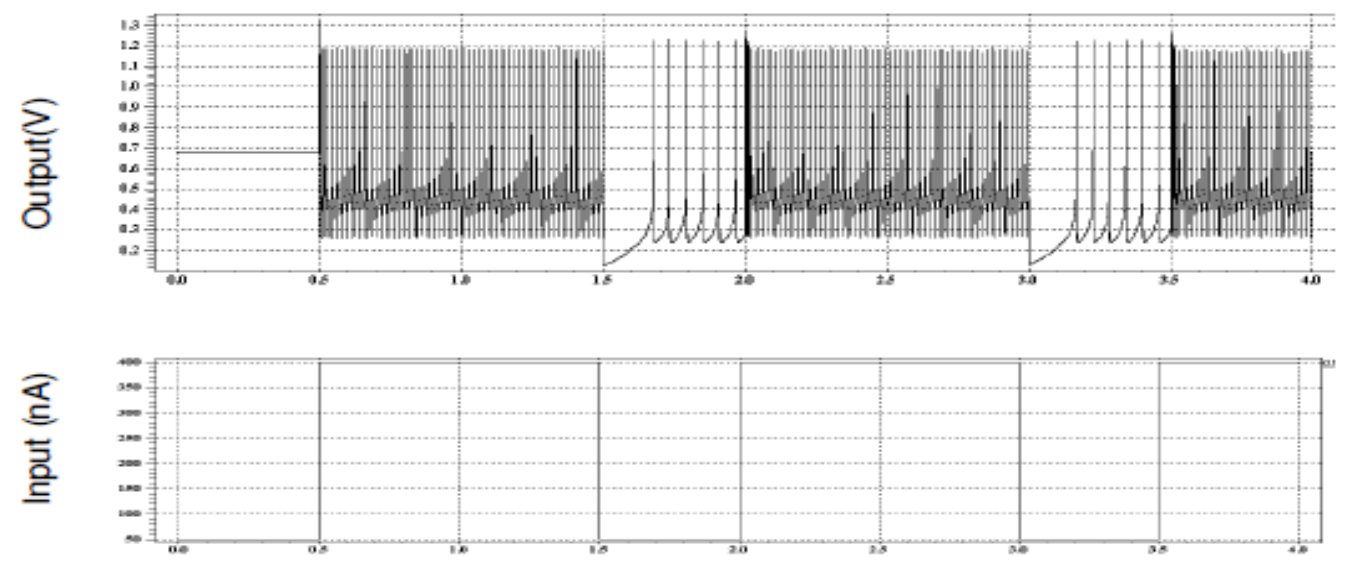

time (ms)

Fig 3.9 Response of amplitude variation on the fast spiking (FS2) of cortical neuron

Another spiking pattern (FS2 and RS2) for different amplitude and different control voltage is studied. At the input side of Cortical neuron step of 400nA and 50nA are given with the threshold voltage $1.4 \mathrm{~V}$ and supply voltage $3 \mathrm{~V}$. It is observed that for $400 \mathrm{nA}$ the frequency of spiking pattern is high while the spike frequency of spiking pattern for $50 \mathrm{nA}$ is $10 \mathrm{KHz}$. For both the input control voltage $\mathrm{Vd}$ is set to $1.1 \mathrm{~V}$.

It is also observed that delay for lower amplitude is more than that for higher amplitude. The delay for $400 \mathrm{nA}$ is $0 \mathrm{~ms}$ while delay for $50 \mathrm{nA}$ is $0.15 \mathrm{~ms}$.

It is observed that, if amplitude of input is increased more spikes are needed to fire the circuit, slow variable capacitor doesn't get sufficient time to discharge results in the fast spiking and if decrease the amplitude less spikes are needed to fire the circuit results towards the regular spiking keeping the Vd constant for both the magnitude.

\section{CONCLUSION}

The CMOS Neuron circuit presented is capable of generating spiking and bursting firing behaviors with a biologically plausible spike shapes. The circuit behavior has been verified via spice simulation. The single circuit mimics most of the electrophysiological cortical neuron types and is capable of producing a variety of different behaviors, with diversity similar to that of real biological neuron cells. The behavior of this universal cortical neuron cell can be adjusted by using only one external biasing voltage. The circuit is implemented using only 14 MOSFETs and occupies a small silicon area. Hence the circuit provides simple, compact and easily configurable cortical neuron for building massively parallel analog neuromorphic networks that closely resembles the circuit of neocortex. It is also possible to obtain each type of neuron with different characteristics (frequency of spiking \& accommodation) by changing the width/length $(\mathrm{W} / \mathrm{L})$ ratio of the transistors M4 of membrane circuit and M6, M7of slow variable circuit. The circuit is capable of representing a wide variety of cell types, and with required accommodation and firing frequency by switching (W/L) of the key transistors. The circuit is designed using $1.25 \mathrm{um}$ technology. Apart from implementing different types of neuron, this circuit also enables the 
International Journal on Soft Computing ( IJSC ) Vol.2, No.4, November 2011

designing of a variety of different behavioral cell clusters in each cortical neuron types, with diversity similar to that of biological neuron cells. The variety of behavior is obtained in a single circuit only requiring changing one bias voltage. This voltage can be set externally or could be stored/controlled locally enabling dynamic switching of spiking modes and characteristics.

\section{REFERENCES}

[1] Hsin Chen, Sylvain Saighi, Laure Bahry and Sylvie Renaud,"Real Time Simulation of Biologically Realistic Stochastic Neurons in VLSI", IEEE Transaction on Neural Networks, Vol 21, No. 9, September 2010.

[2] Hongbo Jia,, Nathalie L.Rochefort, Xiaowei Chen and Arthur Konnerth, "Dendritic Organization of Sensory Input to Cortical Neuron in Vivo", Vol 464/29 April 2010/doi:10.1038/nature 08947.

[3] Jayawan H.B Wijekoon, Piotri Dudek, "Compact Silicon Neuron Circuit with Spiking and Bursting Behaviours”, Science Direct, Neural Networks 21 (2008) 524-534.

[4] Chiara Bartolozzi and Giacomo Indiveri, "Synaptic dynamics in analog VLSI", Neural computation $19,2581-2603(2007)$

[5] Jayawan H.B.Wijekoon and Piotr Dudek, "Spiking and bursting firing pattern of a compact VLSI Cortical neuron circuit", International joint conference on Neural-Network, Oriando, Florida, USA, Aug 12-17,2007

[6] Mircea I.Chelaru and Valentin Dragoi, "Asymmetric synaptic depression in cortical networks", Cerebral cortex advance access published August 9,2007.

[7] Richard B.Wells, "Cortical Neurons and Circuits, A Tutorial Introduction”, April 2005.

[8] G.Indiveri, "A low power adaptive integrate-and-fire neuron circuit." IEEE Int.Symp.circuits and systems, ISCAS, pp IV820-823,2003.

[9] Y.Kanazawa, T.Asai and Y.Amemiya, "A hardware depressing synapse and it's application to contrast-invariant pattern recognition”.vol 102,no.729(NC 2002 134-171) 2003 Japan

[10] Shih-Chii Liu, “Analog VLSI circuits for short-term dynamic synapses”, EURASIP journal rev. 25sep-2002.

[11] S.R.Schultz and M.A.Jabri, “ Analogue VLSI integrated-fire neuron with frequency adaptation",Electronic Letters, vol.31, no.16, pp.1357-1358, Aug 1995.

[12] R.Douglas, M.Mahowald, and C.Mead, "Neuromorphic analogue VLSI”, Ann.Rev.Neurosci, Vol. 18, pp.255-281,1995.

[13] J.P.Lazzaro, "Low-power silicon axons, neurons, and synapses", in silicon implementation of pulse coded neural networks, m.E.Zaghloul, J.L.Meador, and R.W.New-comb, Eds.,pp.153-164. Kluwer Academic Publishers, Norwell, MA, 1994.

[14] B.L.Barranco, E.S.Sinencio, A.R.Vazquez, and J.L.Huertas, "A CMOS implementation of Fitz-HughNagumo neuron model”, IEEE Journal Solid-State Circuits, Vol.26,pp.956-965,1991.

[15] M.Mahowald and R.Douglas, “A silicon neuron”, Nature, vol.354,no.6354,pp.515-518,1991 